\title{
LA NORME DÉMOCRATISÉE DU FRANÇAIS PARLÉ
}

Dans la variété des méthodologies en linguistique, on observe constamment deux orientations générales qui révèlent la double nature de ce domaine scientifique. D'un côté, la linguistique descriptive a pour fonction de dire ce que la (ou une) langue est; d'un autre côté, la réglementation prescriptive tend à privilégier certains usages linguistiques dans la mission de dire ce que la (ou une) langue doit (ou devrait) être. C'est dans les deux acceptions de ces approches qu'il est nécessaire de concevoir la définition de la norme linguistique.

\section{La norme idéale et l'usage}

Les rapports entre la norme et l'usage d'une langue font partie de un large complexe des relations entre un système linguistique - en tant que structure idéale de règles d'énonciation et inventaire de formes acceptables - et l'usage flexible (parole) de ces consignes. La stratification du code linguistique est destinée à la formation individuelle et multipliable des modes d'expression; cela signifie, entre autres choses, que le code doit fournir un cadre formel, solide et synchroniquement constant, pour la compétence linguistique que les locuteurs acquièrent progressivement durant le procès d'apprentissage. Le code linguistique est un réseau structurel dont la maîtrise permet essentiellement à tous les membres d'une communauté linguistique de communiquer avec succès. Il est donc nécessaire que le code communicatif soit structurellement idéalisé, puisque les représentations mentales de ses usagers sont nécessairement formelles. Cependant, les actualisations individuelles de ce code et les perceptions des résultats de ces choix linguistiques sont irrévocablement réelles et flexibles, toujours accordées aux réalités des situations communicatives spatio-temporelles et à la compréhension des contextes extra-énonciatifs.

La nature communicative du comportement communautaire de l'homme, d'un autre côté, témoigne d'une forte fonction sociale d'un système linguistique. L'idéalité du code peut donc également être considérée comme une sorte d'orientation de l'usage. C'est là que le système communicatif prend les premières formes d'une norme: l'usager est censé l'utiliser s'il veut que ses productions linguistiques soient socialement acceptables, que, dans une situation déterminée, elles soient convenables, et/ou qu'elles soient énoncées selon les règles de correction, donc: normales. Dans les processus de production et de perception des messages linguistiques, le locuteur est donc plus ou moins conscient de communiquer avec la norme. 


\subsection{La double définition de la norme linguistique}

La norme linguistique peut se définir sous deux angles méthodologiques radicalement différents.

Du point de vue prescriptif, celui du «bon usage», la norme est un système de consignes qui régissent le choix correct des moyens linguistiques si leur usage se veut accordé à un certain idéal esthétique ou social. Cela veut dire aussi que la norme tend à interdire l'emploi de certains moyens qui empêcheront le locuteur d'atteindre cet idéal; dans ce sens, le purisme et l'intolérance linguistiques peuvent se manifester fréquemment, mais toujours comme de simples corrélations à ces proscriptions.

Du point de vue descriptif, la norme est ce qui est d'usage commun et général à l'intérieur d'une communauté linguistique déterminée. La langue étant considérée comme une institution humaine, la norme linguistique peut se comprendre sous forme d'une institution sociale, mettant en place les principes de l'expression linguistique standard 1 .

La dynamique des principes d'institution et de fonctionnement de la norme linguistique ouvre une question essentielle, concernant les changements de la norme et ceux de l'usage: dans quel sens vont les influences, lequel des deux (norme ou usage) est capable de modifier l'autre? Il est certain que les éléments prescriptifs de la norme linguistique tendent à diriger le comportement de l'usage dans certaines situations communicatives (plus ou moins prévisibles); c'est là dedans qu'il faut chercher l'essentiel de la fonction sociale d'un système linguistique. Mais il n'est pas moins révélateur d'observer les tendances selon lesquelles l'usage réagit à la réglementation normative.

Toute norme, imposée dans une synchronie, est idéalisée et apparemment inaltérable. Elle se manifeste en tant que repère réglementaire à l'usage linguistique socialement accepté, mais elle peut également servir à représenter l'arbitraire des systèmes linguistiques en général. L'évolution d'une langue n'est rien d'autre qu'un enchaînement continu de synchronies déterminées - donc: temporairement formalisées et fonctionnellement discontinues - ce qui rend difficile la stricte définition de leurs limites temporelles. L'examen des diverses étapes évolutives d'une langue donnée montre irrésistiblement que le schéma des règles systématiques se transforme selon la progression de l'usage dans le temps. Il est donc essentiel de comprendre le développement des systèmes linguistiques à travers la dynamique de leurs usages quotidiens et à travers la communication des usagers avec les règles en vigueur.

\subsection{La responsabilité de la norme envers les spécificités des usages}

L'acquisition plus ou moins intentionnelle de la norme et le respect conscient de l'acquis normatif dépendent de la stratégie individuelle de chaque locuteur particulier. C'est une stratégie dont il peut se servir consciemment selon ses intérêts et objectifs

1 Dans la terminologie normative française, la notion de «langue standard» a été élaborée par Pierre Léon dans son ouvrage fondamental Prononciation du français standard (1966). 
sociaux. Il n'est pas moins important de prendre en considération le rapport inconscient que le locuteur entretient avec la norme linguistique. La délimitation spatiotemporelle de tout système normatif unifié peut être ressentie par les locuteurs comme l'institution d'un réseau structurel qui, à l'intérieur de leur langue, détermine ce qui est idéal, neutre, socialement non-marqué ${ }^{2}$ ou - tout simplement - correct. Ce dernier jugement de l'individu parlant sur les propriétés normatives de son propre usage émane du fait que la norme linguistique est en effet considérée par lui comme une prescription; c'est par là que le locuteur crée, à travers ses activités communicatives, la relation implicite vis-à-vis d'une norme socialement explicite. L'individu parlant forme parfois sa propre norme individuelle qui peut, à travers son jugement de la fiabilité de celle-ci par rapport à la «norme systémique», s'avérer fictive. ${ }^{3}$

Dans la communication, la norme est toujours présente par sa fonction de «correcteur d'expression» qui impose incessamment au locuteur le cadre pour une sorte d'(auto)censure orale ou écrite. Il arrive que, au cours du processus de l'énonciation spontanée, le locuteur s'écarte des règles (prescrites pour telle ou autre situation communicative) et commet par là ce que la norme standardisée désigne comme faute, ou encore comme un usage mauvais, inadmis ou même interdit.

«Alors, qu'est-ce qu'on a besoin ... euh, de quoi est-ce qu'on a besoin pour expliquer cette argumentation?» disait le célèbre professeur Oswald Ducrot lors de l'une des conférences qu'il a données en 1997 à l'Institut des Sciences Humaines à Ljubljana. Le linguiste était en train de défendre son hypothèse sur une spécificité argumentative. En situation formelle de conférence, il lui est arrivé d'employer une structure syntaxique incovenable non seulement par rapport aux règles systémiques d'utilisation d'une périphrase verbale indirectement transitive «avoir besoin», mais

2 L'usage «correct» de la norme linguistique peut se présenter comme socialement non-marqué dans les milieux culturels où le passage bilatéral entre le système des prescriptions normatives et l'usage flexible dans des situations de communication quotidienne est relativement équilibré. Dans ce cas, un tel usage ne paraît pas trop éloigné de l'image idéale (systémique) da la langue utilisée. De ce point de vue, il est intéressant de considérer cette relation dans la communauté linguistique slovène. Abstraction faite des marques dialectales extrêmement fortes et variées, l'usage informel coïncide très rarement avec la norme idéalisée, et cela à tous les niveaux structuraux de l'expression linguistique: phonologique, morphosyntaxique et lexical. Les réalisations intentionnellement normatives sont loin de passer pour non-marquées; elles risquent de paraître prétentieuses. C'est d'ailleurs un écart structurel qu'on peut mesurer même dans des situations normativement très déterminées: un grand nombre, sinon la majorité des locuteurs médiatiques professionnels laissent percevoir dans leurs actualisations prétendûment normatives les marques inconscientes qui font reconnaître facilement leur provenance sociale ou géographique. Sur une population linguistique de deux millions, cette spécificité paraît d'autant plus évidente.

3 A. Borrell, M. Billières (1989), str. 58: «...il convient d'opérer la distinction entre les «normes systémiques», purement linguistiques, et les "normes fictives", qui font appel à l'wimaginaire linguistique» des locuteurs, donc d'ordre psychologique. Dans certains cas, ces "normes fictives" influent sur les "normes systémiques" soit dans le sens d'une évolution soit comme un frein.» Cette conception des différences entre les phénomènes normatifs indique une nette différenciation entre les niveuax «objectif» et "psychologique» dans la formation de la norme. On dira aussi que le premier niveau normatif est «explicite» et l'autre «implicite», puisque le locuteur entretient avec les deux des rapports plus ou moins conscients, et puisque le premier est formulé socialement, l'autre individuellement. 
surtout par rapport à la situation donnée. Certes, Ducrot savait très bien quelle était la structure qu'il eût été convenable d'utiliser. S'il y avait pensé, il aurait très bien pu harmoniser son énoncé avec la norme et avec sa situation extralinguistique, mais il a tout de même dit autre chose. Pourquoi, exactement, a-t-il commis cette «faute»?

La flexibilité de la perception permet, en des situations communicatives moins formelles (c'est-à-dire les plus fréquentes), des productions linguistiques non-idéales par rapport à la norme. Il est d'ailleurs possible et attesté que les locuteurs du français utilisent «qu'est-ce qu'on a besoin» dans la formation normativement non-contrainte de leurs messages oraux. Il est évident que, dans une situation qui exige du locuteur l'emploi du registre normalisé - et donc la forme «de quoi est-ce qu'on a besoin» - le conférencier a laissé son mode énonciatif s'échapper sur le territoire de l'usage quotidien. Oswald Ducrot est un locuteur francophone très habile; cela signifie que l'usage linguistique spontané peut exercer, même chez les meilleurs «maîtres» de l'énonciation, une forte influence sur les messages plus ou moins réfléchis normativement. La première partie «déplacée» de l'énoncé de Ducrot révèle donc un «mauvais usage» inconscient, suivi d'un "corrigé», celui-ci étant sans aucun doute le fruit d'une réflexion consciente. Ce corrigé se manifeste comme un moyen d'énonciation stratégique que le locuteur investit dans l'effort d'approprier son énoncé au niveau acceptable pour la situation communicative donnée.

Cet exemple n'explique pas quels sont les écarts non-normatifs que la norme pourrait (ou devrait) inclure dans ses schémas. Il indique tout simplement que les procédés inconscients dans les actualisations (et perceptions) énonciatives révèlent et peuvent perpétrer des écarts plus typiques et plus généraux par rapport à la norme explicite et en dernière instance, par rapport au système linguistique lui-même. Comme les systèmes de représentations orthographiques réagissent au développement de l'expression orale dans le temps, c'est l'usage généralisé moyen qui détermine à long terme la dynamique, la flexibilité et les changements des systèmes normatifs.

Le dilemme final de la linguistique normative peut paraître trop général, mais n'en est pas pour autant moins complexe: quel doit être, dans une période évolutive donnée, le rapport entre l'équilibre, l'arbitraire, la flexibilité et la perméabilité d'un système normatif, et son usage réel, et quels doivent être les critères selon lesquels les écarts typiques se mettent en valeur, pour que la structure de la norme linguistique puisse raisonnablement répondre à la langue que créent dans le temps ses utilisateurs? On met ici à l'épreuve toute une compréhension de la spontanéité des comportements linguistiques et de leur développement continu. La dynamique des normes linguistiques pose des questions auxquelles la linguistique n'est pas invitée à réagir par la fabrication des solutions idéales, classificatoires et arbitraires. Au contraire; du côté analytique, elles demandent à la linguistique la recherche et l'interprétation des relations linguistiques données, du côté politique (dans l'acception la plus large du terme), elles l'incitent à ouvrir sur la réalité des prédictions évolutives et bien fondées concernant les formes possibles d'une cohabitation de la norme et de l'usage. 


\section{Le développement de la norme du français parlé}

La dualité déterminante du fonctionnement de la norme linguistique sera ici «illustrée» par l'exemple de la normativisation du français parlé, et ensuite par les implications contemporaines du discours médiatique.

L'histoire de la norme du français parlé se dessine surtout selon les critères géographiques et sociaux, caractéristiques de la principale communauté francophone en Europe. Un premier aspect historique est sans doute l'antagonisme entre les faits linguistiques que la philologie romane désigne sous les notions de langue d'oc (sud) et de langue d'oül (nord). Il est important de considérer que la France méridionale, en comparaison avec les régions septentrionales, a connu une bien plus grande différenciation idiomatique dont les conséquences (et les richesses) se font encore sentir dans la synchronie linguistique qui est la nôtre. Il était donc inévitable que l'initiative de la normativisation vînt du nord; il est pratiquement inutile de préciser que la région de l'Ile-de-France, économiquement et politiquement supérieure, ait son ébauche au nord, linguistiquement plus unifié.

On considère que le développement de la norme française s'ouvre en 1539 avec l'Ordonnance de Villers-Cotterêts, par laquelle François I $^{\text {er }}$ impose le francien (parler de Paris et de ses environs) dans les actes officiels et de justice. Le francien devient donc officiellement français en conséquence d'une décision politique centraliste.

\section{1 «Le français parisien cultivé»}

On insiste ici sur le fait que la dynamique des changements de la norme linguistique est étroitement liée au développement de la société par laquelle la langue est utilisée. À l'époque du français classique, la conception du «bon usage» n'occupait pas uniquement les grammairiens. Elle était réfléchie par un grand nombre d'intellectuels désireux de formuler les relations sociales et les ressorts de leur propre activité publique. Au XVII ${ }^{e}$ siècle, le débat sur les questions linguistiques était mené dans divers salons parisiens et dirigé par Vaugelas qui, dans ses célèbres Remarques sur la langue française (1647), définissait la norme du français comme «la façon de parler de la partie la plus saine de la Cour». Ce jugement de valeur, si explicite, désigne le «bon usage» comme une catégorie qualitative, exclusivement caractéristique pour une étroite classe sociale supérieure. Le comportement de l'aristocratie française servait d'exemple à une autre classe sociale, nettement plus nombreuse, celle de la haute bourgeoisie qui s'appropriait, par imitation, les insignes aristocratiques extérieures en tant qu'emblèmes de succès et d'influence sociale. L'ascension de la haute bourgeoisie parisienne a étendu l'application de l'usage considéré normatif sur un cercle plus large, mais toujours relativement restreint des usagers du français. Et encore, d'un autre point de vue, il ne faut pas oublier que la grande plupart des définitions de la norme linguistique à l'époque se fondaient généralement sur les descriptions et jugements qualitatifs de l'écrit, et non de l'oral. 
La centralisation du pouvoir en France est un processus historique qui tend à décroître seulement après la Seconde guerre mondiale. Le réflexe centralisateur contribuait sans cesse à l'affermissement de la position normative du français parisien cultivé. En expliquant cette définition de la norme orale en français, Fouché ${ }^{4}$ soutient que «tous les Français - consciemment ou inconsciemment - tiennent Paris pour exemplaire en ce qui concerne les questions linguistiques ou autres». Il avance également que l'usage linguistique populaire, même en capitale, ne peut pas être tenu pour normatif; que le «français normal» ne peut donc être parlé que par les ressortissants cultivés de la bourgeoisie parisienne.

Dans son analyse de la prononciation française contemporaine ${ }^{5}$, basée sur une enquête linguistique parmi les officiers français en 1945, Martinet avance que plusieurs variantes équivalentes d'une même langue cohabitent sur un large territoire linguistique du français. Son étude montre que ces variantes se distinguent selon les modes de prononciation, et aussi selon les spécificités des structures phonologiques; ce que la phonétique normative décrit comme le français parisien cultivé, ne serait qu'un segment partiellement marqué ${ }^{6}$ de la totalité des usages linguistiques français.

\section{2 «Le français standard»}

Au terme de la Seconde guerre mondiale, la France a connu une démocratisation générale des rapports sociaux. Le relâchement de l'espace politique avait pour conséquence un épanouissement inédit des activités médiatiques. Parallèlement, c'est l'époque où les linguistes ont commencé à s'intéresser plus fermement à la parole: c'est le début d'une relativisation progressive de la tradition saussurienne qui est fondée sur l'analyse exclusive du système de la langue. Dans ces circonstances sociales et scientifiques, la recherche de la norme parlée française s'est radicalement démocratisée en débouchant sur l'observation des réalités dans les manifestations linguistiques orales. L'orientation vers une tolérance objective par rapport à la spontanéité de l'expression linguistique n'a depuis cessé de préserver sa fraîcheur scientifique. L'attention des linguistes «normativisants» n'est désormais plus uniquement occupée par la prescription des règles systématiques: l'analyse des actualisations linguistiques spontanées qui créent la norme sociale prend de l'envergure.

Le concept normatif du français standard a été minutieusement élaboré par Pierre Léon qui, se fondant sur les enquêtes largement conçues, constate que la communauté linguistique a pris une certaine distance vis-à-vis du français parisien cultivé et qu' «il existe une prononciation standard dont le niveau moyen est grosso modo représenté

4 P. Fouché (1936), pp. 201-202.

5 Cette étude a été publiée postérieurement, dans A. Martinet (1971).

6 Marqué dans le sens de supériorité et de prétention; ces deux qualificatifs du français parisien cultivé seraient ressentis et adjugés par la couche sociale française moyenne, majoritaire et non-parisienne, donc la majorité des utilisateurs du français à l'époque où commence à se former l'image «démocratique» du français standard. 
par les annonceurs et les interviewers de la radio...; dans l'ensemble, leur prononciation reflète l'usage moyen, sans recherche (pour plaire au grand public) et sans familiarité excessive (à cause du micro). De toute façon, c'est le modèle proposé à longueur de journée à des millions de Français et c'est celui qui a le plus de chances de triompher un jour.» ${ }^{7} \mathrm{~L}$ 'analyse de Léon est la première à avoir considéré le fait que les médias parlants peuvent jouer un rôle crucial dans la formation d'une prononciation qui serait linguistiquement typique pour une proportion absolument majoritaire de la communauté francophone. Il insiste aussi sur la description du «niveau moyen» de la prononciation standard qui, sous l'influence du discours médiatique, se développe plus rapidement dans les milieux urbains. Ce standard parlé a également été relativement vite approprié par la campagne française, principalement à cause des médias, mais aussi à cause du ressentiment psychologique toujours existant envers la position centrale et privilégiée de Paris.

\section{3 «Le français standardisé»}

Dans les années 60 et $70 \mathrm{du} \mathrm{XX}^{\mathrm{e}}$ siècle, le français parisien cultivé, tel qu'il a été conceptualisé depuis trois cents ans, a définitivement perdu son prestige normatif. C'est surtout dans les débuts du processus de la décentralisation du système politique et social français que la grande plupart des utilisateurs non-parisiens du français le qualifiaient de «prétentieux» ${ }^{8}$, et cela non seulement à la campagne, mais aussi dans les milieux urbains de province, surtout au sud. Les locuteurs méridionaux, sans doute marqués de leur évidente spécificité dialectale, ont très vite identifié le français standard (médiatisé) comme normatif et comme «neutre», pour le substituer aussi efficacement que possible contre la norme parisienne. Dans les dernières décennies, un autre phénomène très important a contribué à la standardisation de la prononciation française, lui aussi déclenché par la décentralisation: l'augmentation considérable de la mobilité géographique de la population. C'est une tendance migratoire interne qui est encore aujourd'hui largement observable en France hexagonale, même si elle connaissait une légère décroissance dans les années $90.9^{9}$ Dans les circonstances du chômage réel ou potentiel, les jeunes surtout décident très fréquemment de prendre des postes de travail en dehors de leurs lieux de naissance ou de scolarité. On n'observe donc pas uniquement l'affluence traditionnelle des jeunes (diplômés) de campagne vers Paris; la mobilité géographique des employés dans toutes les régions françaises (et leurs centres) a fait que les gens de différentes provenances françaises continuent à se mélanger surtout dans les agglomérations urbaines.

On peut donc affirmer que le français standard a fini par perdre les derniers traits de la stricte prescription qui persévérait longuement en tant qu'héritage du français

\footnotetext{
7 P. Léon. (1968), p. 69.

8 P. Léon (1983), p. 16.

9 A. Borrell, M. Billières (1989), pp. 59-60.
} 
parisien cultivé. L'initiative normativisante est maintenant cédée à l'usage linguistique démocratisé - et largement défini - qui peut ainsi créer son propre modus vivendi social. Le français standard accepte dans son cadre toutes les caractéristiques de l'expression linguistique qui permettent au locuteur de parler d'une façon non-marquée, voire «non-remarquée», sur la totalité du territoire de la francophonie hexagonale. Bref, c'est une norme «neutralisée» qui, dans la formulation démocratique (i.e. nonprestigieuse) du standard, permet de produire à tout le monde des messages oraux dont le ton ne sera plus chargé d'aucun privilège social. C'est le type de norme parlée que $\mathrm{H}$. Walter et A. Martinet définissent très clairement: «Les bonnes prononciations sont celles qui passent inaperçues, les mauvaises, celles qui soudain vous rappellent, ne serait-ce qu'à un niveau très inférieur de la conscience, que votre interlocuteur est de telle origine, nationale, géographique ou sociale.» ${ }^{10}$ Dans les diverses situations communicatives, les messages oraux normativement bien équilibrés permettent donc au locuteur de n'attirer aucune attention circonstancielle concernant l'expression parlée ou le mode de sa formation.

Il est certain que la formation de la norme parlée est un processus intrinsèquement dynamique. Son dynamisme et sa variabilité actuels s'accélèrent au rythme de l'expansion extrême des médias parlants qui régissent de plus en plus ses modifications. Comme ces médias (radiodiffusion et télévision) deviennent omniprésents ${ }^{11}$ dans la vie de toute communauté linguistique moderne et comme l'influence de leur rapidité et multi-orientation est décisive, certains auteurs définissent la forme normative du français parlé sous terme plus concis de «français standardisé». ${ }^{12} \mathrm{C}$ 'est une notion qui est conçue avec plus de précision justement par ce qu'elle implique la dynamique temporelle ${ }^{13}$ du processus normatif. Il n'est pas sans importance de préciser que les activités médiatiques contribuent d'une façon considérable à la démystification de certains phénomènes sociaux. Si donc avant les années $60 \mathrm{du} \mathrm{XX}^{\mathrm{e}}$ siècle, le français parisien cultivé n'avait aucune difficulté concurrencielle à passer pour privilégié entre les seg-

10 A. Martinet, H. Walter (1973), p. 17.

11 Il importe de comprendre cette «omniprésence» médiatique à travers les principes de son fonctionnement linguistique. La communication médiatique est, à savoir, une communication généralement unilatérale. Cela veut dire qu'un locuteur médiatique entre en rapport communicatif avec une multitude d'《interlocuteurs» qui n'ont pas la possibilité de participer simultanément au temps de la communication. Autrement dit: ils ne deviennent jamais (ou très rarement) coénonciateurs ou co-locuteurs. Ils sont donc soumis à la simple perception plus ou moins attentive d'une multitude de messages oraux auxquels il ne donnent aucune réponse. La structuration spécifique des messages oraux médiatiques, ainsi que la nature de leur occurrence, exercent donc nécessairement une influence sur les niveaux conscients (normativisants) et/ou inconscients de la compétence linguistique du récepteur. - Cela signifie, évidemment, que la démocratisation médiatique n'exclut en rien l'éventualité d'une autocratie linguistique.

12 A. Borrell, M. Billières (1989), p. 57.

13 Dans la même orientation terminologique, on préfère dans l'analyse de l'énonciation le terme d'uactualisation» à celui de «réalisation», puisqu'il tient mieux compte de la situation spatio-temporelle de toute production linguistique. 
ments de l'usage francophone, on peut conclure que le statut de la norme parisienne, prestigieuse aux oreilles de la majorité hors-capitale, pouvait uniquement être balayé par une démocratisation bien déterminée de l'expression linguistique. Autrement dit: la brume mystificatrice autour du «français parisien cultivé» n'a pu s'évaporer que par l'exercice oral d'une dictature médiatique généralisée.

\section{Bibliographie}

BLANCHE-BENVENISTE, Claire (1991): Le français parlé. Études grammaticales, coll. Sciences du langage, Éditions du CNRS, Paris.

BORRELL, André, BILLIĖRES, Michel (1989): L'évolution de la norme phonétique en français contemporain, in: La Linguistique 25/2, pp. 45-62.

FOUCHÉ, Pierre (1936): Les diverses sortes de français au point de vue phonétique, in: Français moderne 4, pp. 199-216.

FOUCHÉ, Pierre (1959): Traité de prononciation française, Klincksieck, Paris.

LÉN, Pierre (1966): Prononciation du français standard, Didier, Paris.

LÉON, Pierre (1983): Dynamique des changements phonétiques dans le français de France et du Canada, in: La Linguistique 19 , pp. 13-28.

LEON, Pierre (1992): Phonétisme et prononciations du français, coll. Nathan Université, Nathan, Paris.

MARTINET, André (1971): La prononciation du français contemporain, Droz, Paris.

MARTINET, André, WALTER, Henriette (1973): Dictionnaire de la prononciation française dans son usage réel, - France-Expansion, Paris.

VITEZ, Primož (1997): Accent $d$ intensité et action intonative en français moderne, in: Linguistica XXXVII, Ljubljana, pp. 71-80.

VITEZ, Primož (1999): Le dynamisme accentuel dans le discours médiatique français, in: Linguistica XXXIX, Ljubljana, pp. 99-121.

WALTER, Henriette (1976): La dynamique des phonèmes dans le lexique français contemporain, France Expansion, Paris. 


\section{Povzetek}

\section{DEMOKRATIZIRANA NORMA FRANCOSKEGA GOVORA}

Jezikovno normo opredeljujeta dve različni izhodišči. S predpisovalnega vidika je norma sistem navodil za pravilno izbiro jezikovnih sredstev, če naj bo njihova raba v skladu $\mathrm{z}$ določenim estetskim ali sociokulturnim idealom. To hkrati pomeni, da si norma prizadeva prepovedati rabo jezikovnih sredstev, ki onemogočajo dosego tega težko opredeljivega socialnega jezikovnega ideala. $Z$ vidika opisnega jezikoslovja pa je norma tisto, kar je v skupni in splošni rabi znotraj določene jezikovne skupnosti. Ker torej velja, da je jezik človeška institucija, potem je jezikovno normo mogoče razumeti kot socialno institucijo, ki vzpostavlja načela standardnega jezikovnega izraza.

Nezavedni procesi v govornih aktualizacijah sčasoma povzročajo tipične in splošne odklone od eksplicitnih norm (ali eksplicitnih jezikovnih sistemov nasploh), ki svoje predpise slej ali prej prilagajajo prav prevladujočim realnim pojavnostim v aktualizacijah. Kakor so se razni načini grafične reprezentacije govora že od nastanka naprej sproti odzivali na spreminjajoče se značilnosti govornega izraza, tako je v reprezentativnih segmentih jezikovnih skupnosti prevladujoča raba jezika tista, ki na vseh jezikovnih ravneh brez izjeme dolgoročno uravnava nastanek, prožnost in razvoj normativnih sistemov.

Zgodovinski pogled na procese oblikovanja normativnosti francoskega jezika razkriva, da med njihovimi razvojnimi dejavniki najpomembnejše mesto zavzemajo geografske prvine in družbenopolitična razmerja, značilna za razvoj frankofonske skupnosti. Za začetek razvoja normirane francoščine šteje leto 1539 , ko je francoski kralj Franc I. s političnim dekretom (torej z močno centralizacijsko potezo) državi določil obvezno uporabo narečja, kakršega so govorili na območju Ile-deFrance, torej v Parizu z okolico. Ta odločitev je imela daljnosežne jezikovnopolitične posledice, saj je t.i. "kultiviran pariški govor" v dolgo trajajočih zgodovinskih razmerah centralizirane francoske družbe vse do druge polovice XX. stoletja splošno veljal za normirano varianto francoskega govora.

Prestižni normativni mit o kultiviranem pariškem govoru se je začel razblinjati šele po drugi svetovni vojni, ko je Francijo prvič zajel val decentralizacijskih političnih pobud. Pariška norma je zunaj Pariza hitro obveljala za "pretenciozno", proces demokratizacije govorne norme pa se je bliskovito pospešil $\mathrm{z}$ razmahom govornih medijev, posebej televizije. Zaradi močnega in splošnega vpliva medijskega govora na jezikovno rabo (in posebej na njene nezavedne segmente) se je $\mathrm{v}$ jezikoslovni terminologiji kmalu pojavil normativni koncept "standardnega francoskega govora". Ker pa je oblikovanje "povprečnega" (odtod: demokratičnega) govora dinamičen proces, ki v zadnjih desetletjih hitreje kot kdaj prej spreminja tudi podobo govorne norme, se je v sodobnem jezikoslovnem izrazju uveljavil še ustreznejši pojem "standardiziran francoski govor", ki natančneje upošteva prostorsko in časovno dinamiko vsakršne govorne aktualizacije. 